\title{
Direct Numerical Simulations of Turbulent Spray Combustion: Behavior of Scalar Dissipation Rate
}

\author{
By Abouelmagd Abdelsamie* \\ Dominique Thevenin ${ }^{\dagger}$
}

Scalar dissipation rate is an essential parameter in combustion theory; understanding its behavior is necessary for developing and improving combustion models. In most cases, the scalar dissipation rate shows different trends under different combustion conditions, and it is thus case-dependent. Spray combustion is categorized as a partiallypremixed combustion system, leading to very complex processes. The objective of this work is to investigate the behavior of scalar dissipation rate in turbulent spray combustion using Direct Numerical Simulations (DNS). For this purpose, a temporally-evolving jet (TEJ) configuration has been examined. 3D simulations have been conducted using the inhouse code DINO. This configuration constitutes a perfect numerical setting to investigate scalar dissipation rate, mixing, and shear in spray combustion. The conditional mean of scalar dissipation rate shows a non-monotonic behavior in mixture fraction space, highlighting the complexity of the interaction between spray, flame, and turbulence.

Keywords: DNS, Scalar dissipation rate, Spray, Turbulent combustion.

\section{Introduction}

Spray combustion is commonly encountered in many industrial devices, such as gas turbines and diesel engines. Understanding of liquid-fuel combustion is thus necessary to improve fuel efficiency and control emissions of these applications. Spray combustion includes many physical aspects, in particular evaporation and ignition. For a better understanding of such complex phenomena and to develop improved models, detailed investigations are required, typically relying on experiments and/or on Direct Numerical Simulations. DNS have been employed to investigate reactive droplets and spray combustion for almost two decades, considering many aspects and configurations. With growing computer power, different modeling levels have been combined, considering either single-step mechanisms (Mashayek, 2000; Réveillon and Vervisch, 2005; Domingo et al., 2005; Réveillon and Demoulin, 2007; Wandel et al., 2009; Fréret et al., 2011; Fujita et al., 2013; Kitano et al., 2013; Kitano et al., 2014), detailed kinetics in 2D simulations (Wang and Rutland, 2005; Wang and Rutland, 2007; Réveillon et al., 2011), or, more recently, detailed chemistry in 3D simulations (Neophytou et al., 2011; Neophytou et al., 2012; Borghesi et al., 2013; Wang et al., 2014; Jin et al., 2016). Three configurations have been mainly considered: (1) Spray dispersion and ignition in homogeneous conditions and/or in flow with zero mean-flow velocity (Mashayek, 2000; Wang and Rutland, 2005; Réveillon and

\footnotetext{
*University of Magdeburg "Otto von Guericke", Germany.

† University of Magdeburg "Otto von Guericke", Germany.
} 
Demoulin, 2007; Réveillon et al., 2011; Neophytou et al., 2011; Neophytou et al., 2012; Borghesi et al., 2013; Kitano et al., 2014; Wang et al., 2014); (2) Spatiallyevolving jets (Réveillon and Vervisch, 2005; Domingo et al., 2005; Fujita et al., 2013; Kitano et al., 2013; Jin et al., 2016); and (3) Temporally-evolving jets (Wang and Rutland, 2005; Abdelsamie and Thévenin, 2017). Common conclusions of all these references are that the equivalence ratio and droplet size are the most critical parameters controlling spray combustion mode and ignition. Although these studies answered many questions, the impact of different operation conditions and configurations has not been sufficiently clarified yet (Fujita et al., 2013); more specifically, the effect of shear and behavior of scalar dissipation rate are not completely clear. Furthermore, scalar dissipation rate plays an essential role to understand autoignition, and it will determine the final state (extinction/ ignition). Therefore, it is very important to understand the behavior of scalar dissipation rate. This work examines in details the impact of shear on the ignition process and on the scalar dissipation rate using DNS. This article is organized as follows: After the introduction, governing equations are reviewed; then, the numerical settings are presented, followed by results and discussion, before closing with the conclusions.

\section{Governing Equations}

The simulations in this work are performed using the in-house code called DINO (Abdelsamie et al., 2016). In this code, the low-Mach number model is employed to simulate the gas mixture (continuous phase), where the total mass conservation equation reads:

$$
\frac{\partial \rho}{\partial t}+\frac{\partial \rho u_{i}}{\partial x_{i}}=0
$$

and the equation of conversation of each species mass fraction $Y_{\mathrm{k}}$ reads

$$
\frac{\partial \rho Y_{k}}{\partial t}+\frac{\partial \rho u_{i} Y_{k}}{\partial x_{i}}=-\frac{\partial \rho V_{k, i} Y_{k}}{\partial x_{i}}+\dot{\omega}_{k}, \quad \text { for } k=1,2, \ldots, N \text {. }
$$

The momentum conservation equation can be written as:

$$
\frac{\partial \rho u_{i}}{\partial t}+\frac{\partial \rho u_{i} u_{j}}{\partial x_{j}}=-\frac{\partial \tilde{p}}{\partial x_{i}}+\frac{\partial \sigma_{j i}}{\partial x_{j}}
$$

where the viscous stress tensor is:

$$
\sigma_{j i}=-\frac{2}{3} \mu \frac{\partial u_{k}}{\partial x_{k}} \delta_{j i}+\mu\left(\frac{\partial u_{j}}{\partial x_{i}}+\frac{\partial u_{i}}{\partial x_{j}}\right)
$$

In the low-Mach model, the temperature equation simplifies to 


$$
\rho C_{p} \frac{D T}{D t}=-\sum_{k=1}^{N} h_{k} \dot{\omega}_{k}+\frac{\partial}{\partial x_{i}}\left(\lambda \frac{\partial T}{\partial x_{i}}\right)-\rho \frac{\partial T}{\partial x_{i}}\left(\sum_{k=1}^{N} C_{p, k} Y_{k} V_{k, i}\right) .
$$

This set of equations is closed with the equation of state. In Eqs. (1)-(5), $u_{i}$, $T, \rho, \mu, \lambda, Y_{\mathrm{k}}, \dot{\omega}_{k}, \delta_{i j}, C_{p}, C_{p, k}, h_{k}$, and $V_{k, i}$ are $i$-th component of mixture velocity, mixture temperature, mixture density, mixture viscosity, heat diffusivity coefficient, mass fraction of species $k$, net production rate of species $k$, Kronecker delta, specific heat of the mixture at constant pressure, specific heat of species $k$ at constant pressure, enthalpy of species $k$, and the $i$-th component of diffusion velocity of species $k$, respectively. The pressure in the low-Mach number model can be decomposed into two components; thermodynamic pressure, which is computed from the equation of state, and the dynamic fluctuating pressure, $\tilde{p}$ which is computed by solving a Poisson equation. When solving the equation system, two constraints should be retained:

$$
\begin{aligned}
& \sum_{k=1}^{N} Y_{k}=1, \\
& \text { and } \\
& \sum_{k=1}^{N} Y_{k} V_{k, i}=0 .
\end{aligned}
$$

In DINO (Abdelsamie et al., 2016), the droplets are tracked using a pointforce Lagrangian approach (Discrete Particles Simulation, DPS), since droplet diameter is always smaller than grid size (Abdelsamie et al., 2016; Abdelsamie and Thévenin, 2017). Therefore, our simulations can be categorized as DNSDPS. The implemented equations describing droplet location, momentum, mass transfer, and heat transfer read as follows:

$$
\begin{aligned}
\frac{\partial \mathbf{X}_{d}}{\partial t} & =\mathbf{U}_{d}, \\
\frac{\partial \mathbf{U}_{d}}{\partial t} & =\frac{\mathbf{U}_{\infty}-\mathbf{U}_{d}}{\tau_{U, d}}, \\
\frac{\partial a^{2} d}{\partial t} & =-\frac{a_{d}^{2}}{\tau_{a, d}}, \\
\frac{\partial T_{d}}{\partial t} & =\frac{1}{\tau_{T, d}}\left[T_{\infty}-T_{d}-\frac{\mathrm{B}_{T, d} L_{v}}{C_{p, f}^{F} W_{F}}\right],
\end{aligned}
$$

In Eqs. (8)-(11), $\boldsymbol{U}_{d}$ and $\boldsymbol{U}_{\infty}$ are the velocity of the $d$-th droplet and of the surrounding gas at droplet location $\boldsymbol{X}_{d}$. Also, $T_{\infty}, T_{d}, L_{v}, W_{F}, C_{p, f}^{F}$ and $\mathrm{B}_{T, d}$ are mixture temperature in far-field, liquid droplet surface temperature, molar latent heat of droplet vaporization, molar mass of the fuel, specific heat of the fuel vapor in the film region and heat transfer number, respectively. In the film 
region, the properties are computed based on the one-third rule (Abramzon and Sirignano, 1989; Wang and Rutland, 2007; Abdelsamie and Thévenin, 2017) and have the subscript $f$. Three characteristic time scales control the motion and evaporation of the droplets: momentum relaxation time $\left(\tau_{U, d}\right)$, evaporation delay $\left(\tau_{a, d}\right)$, and heating delay $\left(\tau_{T, d}\right)$ :

$$
\begin{aligned}
\tau_{U, d} & =\frac{\rho_{L} a_{d}^{2}}{18 \mu_{f}\left(1+\frac{1}{6} \operatorname{Re}_{d}^{2 / 3}\right)}, \\
\tau_{a, d} & =\frac{\operatorname{Sc}_{d}}{4 \operatorname{Sh}_{d}} \frac{\rho_{L}}{\mu_{f}} \frac{a_{d}^{2}}{\ln \left(1+\mathrm{B}_{m, d}\right)}, \\
\tau_{T, d} & =\frac{\operatorname{Pr}_{d}}{6 \mathrm{Nu}_{d}} \frac{C_{p, L}}{C_{p, f}} \frac{\rho_{L} a_{d}^{2}}{\mu_{f}} \frac{\mathrm{B}_{T, d}}{\ln \left(1+\mathrm{B}_{T, d}\right)} .
\end{aligned}
$$

In these equations, the characteristic times scales are computed as a function of various dimensionless numbers: the droplet Reynolds number $\left(\operatorname{Re}_{d}\right)$, the Spalding mass transfer number $\left(\mathrm{B}_{m}\right)$, and the heat transfer number $\left(\mathrm{B}_{T}\right)$,

$$
\begin{aligned}
& \operatorname{Re}_{d}=\frac{\rho_{\infty} a_{d}}{\mu_{f}}\left|\mathbf{U}_{\infty}-\mathbf{U}_{d}\right|, \\
& \mathrm{B}_{m, d}=\frac{Y_{s, d}-Y_{F, \infty}}{1-Y_{s, d}}, \\
& Y_{s, d}=\frac{W_{F}}{W_{F}+W_{o}\left(p_{\infty} / p_{\text {sat }, d}-1\right)}, \\
& \mathrm{B}_{T, d}=\left(1+\mathrm{B}_{m, d}\right)^{\theta}-1, \\
& \theta=\frac{C_{p, f}^{F}}{C_{p, f}} \frac{\operatorname{Sh}_{d}}{\mathrm{Nu}_{d}} \frac{\operatorname{Pr}_{d}}{\operatorname{Sc}_{d}} .
\end{aligned}
$$

In Eqs. (15)-(19), $Y_{s, d}, Y_{F, \infty}, W_{O}, p_{\infty}$, and $p_{\text {sat }}$ are the vapor surface mass fraction (saturated vapor mass fraction), fuel mass fraction in far-field gas mixture, oxidizer molar mass, far-field pressure and saturated vapor pressure computed with the Clausius-Clapeyron law:

$$
p_{\text {sat }, d}=p_{\text {ref }} \exp \left[-\frac{L_{v}}{\mathrm{R}_{f}}\left(\frac{1}{T_{d}}-\frac{1}{T_{\text {ref }}}\right)\right] \text {, }
$$

where, $\mathrm{R}_{f}, p_{\text {ref }}$, and $T_{\text {ref }}$ are the perfect gas constant, reference pressure, and temperature taken as atmospheric pressure and boiling temperature of the fuel at this pressure, respectively. The latent heat, $L_{v}$ was corrected using the Watson equation, 


$$
L_{v}=L_{v, s}\left(\frac{T_{\mathrm{cr}}-T_{d}}{T_{\mathrm{cr}}-T_{\mathrm{ref}}}\right)^{0.38}
$$

where $L_{v, s}$, and $T_{c r}$ are the molar latent heat at temperature $T_{\text {ref, }}$, and critical temperature of the fuel, respectively. Here the heat transfer number $\left(\mathrm{B}_{T, d}\right)$ depends on the fuel vapor to gas mixture specific heats in the film region $\left(C_{p, f}^{F}\right.$, $\left.C_{p, f}\right)$, on Prandtl number (Pr), Schmidt number (Sc), Sherwood number (Sh), and Nusselt number $(\mathrm{Nu})$, all these quantities being computed similar to Borghesi et al. (2013).

The above equations are spatially discretized in DINO (Abdelsamie et al., 2016) using $6^{\text {th }}$ order central finite-difference method in space and a $3^{\text {rd }}$ order semi-implicit Runge-Kutta approach for temporal discretization. The kinetic and transport properties are computed with the Cantera1.8 library, relying on the skeletal mechanism developed by Patel et al. (2004) to describe n-heptane oxidation, involving $N=29$ species and 52 elementary reactions.

\section{Numerical Setup}

All 3D simulations are performed in a domain with dimensions of $L_{x}=2.4$ $\mathrm{mm}, L_{y}=6.4 \mathrm{~mm}$, and $L_{z}=6.4$, discretized over $96 \times 256 \times 256$ grid points for spanwise, transverse, and streamwise directions, respectively, leading to a fixed grid resolution of $25 \mu \mathrm{m}$. A schematic diagram for this numerical setup is depicted in Figure 1. The domain is periodic in streamwise and spanwise directions, while it has outflow boundary conditions in the transverse direction; these settings are common for TEJ simulations. As it is seen from Figure 1, monodisperse droplets (black spheres) are initially randomly distributed in the central slab (jet region) with a width $H$ of $0.7 \mathrm{~mm}$ and a jet velocity $U_{j}$. The gas domain is filled with oxidizer (air) and has, initially, uniform properties: initial air temperature $T_{\infty, 0}$, and pressure $p_{\infty, 0}$ of $1500 \mathrm{~K}$, and $5 \mathrm{bar}$, respectively. The velocity $U_{\mathrm{co}}$ of the surrounding co-flow is constant for all simulation, at $5 \mathrm{~m} / \mathrm{s}$. The droplets move initially with the same velocity as the surrounding central jet speed and have an initial liquid droplet temperature $T_{d, 0}$ of $300 \mathrm{~K}$, with an initial diameter $a_{d}$ of $10 \mu \mathrm{m}$ while keeping the local equivalence ratio $\phi$ in the central jet at 2 . In all simulations, imposing velocity fluctuations into the central jet region triggers the turbulence. These imposed fluctuations are obtained from well-resolved isotropic turbulent flow fields. The diameters of the droplets always are less than the grid resolution $\Delta x$ and than the Kolmogorov length scale during all simulations, keeping a ratio $a_{d} / \Delta x$ of at least 0.4 , which is an acceptable value for point-force approach in spray combustion (Wang and Rutland, 2007; Neophytou et al., 2011; Wang et al., 2014; Abdelsamie and Thévenin, 2017). 
Figure 1. Numerical Setup

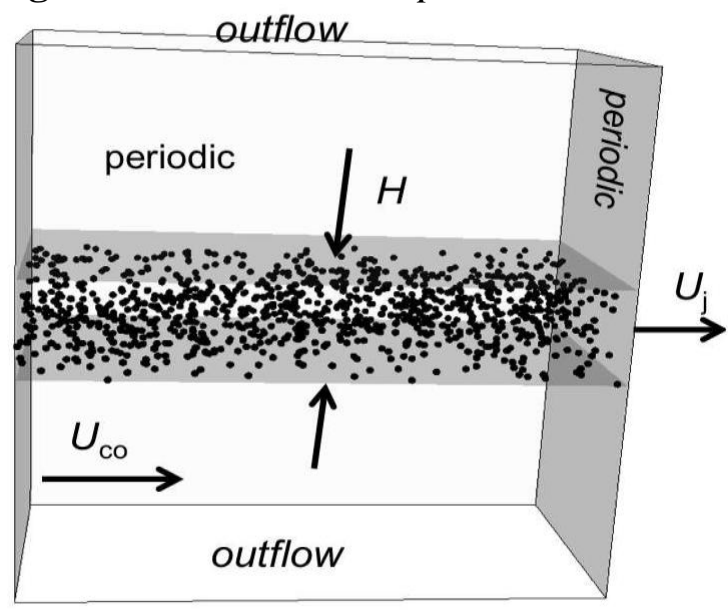

\section{Results}

In this section, four cases will be investigated by changing the jet velocity $\left(U_{j}=25,50,75,100 \mathrm{~m} / \mathrm{s}\right)$ while keeping the co-flow velocity $U_{\mathrm{co}}=5 \mathrm{~m} / \mathrm{s}$, in order to highlight the behavior of the scalar dissipation rate and the impact of shear on the ignition process.

Figure 2. Fuel Spray-turbulence Interaction at $U_{j}=100 \mathrm{~m} / \mathrm{s}$; Red Iso-surface Represents Temperature of $1800 \mathrm{~K}$; Yellow Iso-surface Represents Q-criterion to Reveal Turbulent Structures; Gray Spheres Show the Droplets (Size Multiplied by Factor of 10 for Visualization)

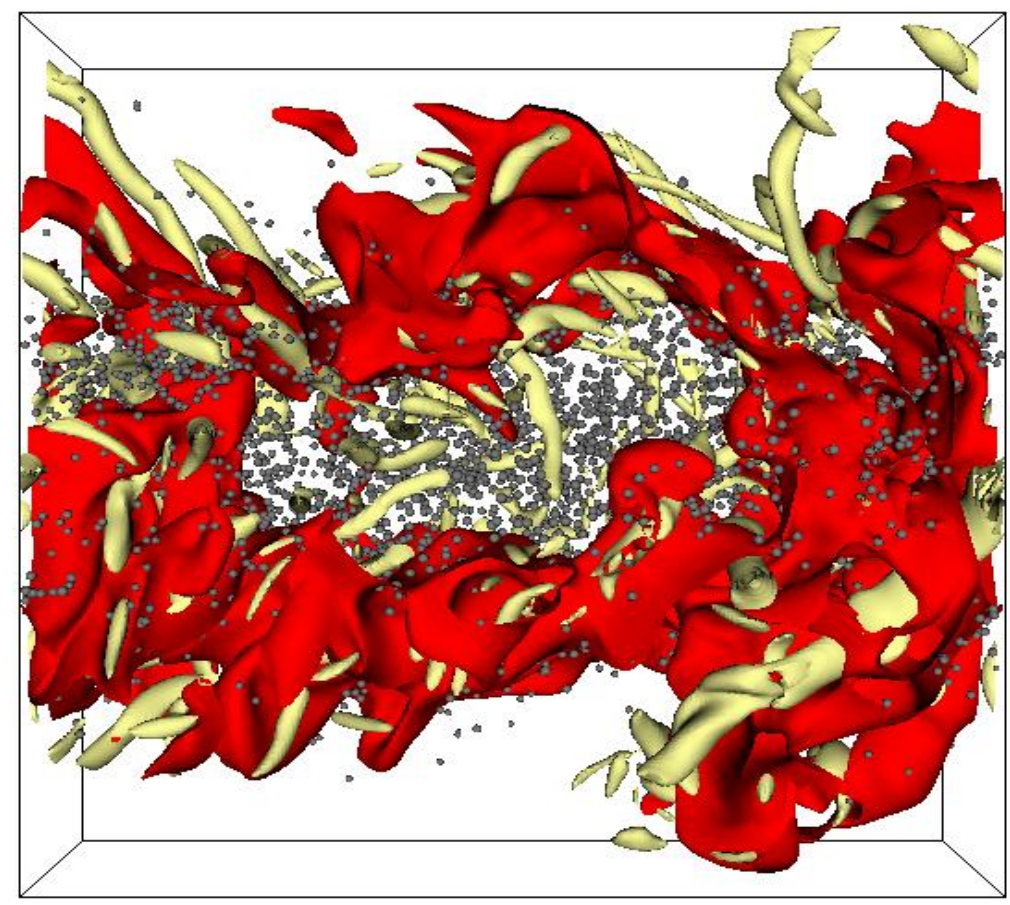


Figure 2 illustrates a typical result obtained when simulating spray in TEJ $\left(U_{j}=100 \mathrm{~m} / \mathrm{s}\right.$ ), a few milliseconds after ignition occurs. In this figure, the gray spheres represent the evaporated droplets (size multiplied by factor of 10 for visualization), the yellow iso-surface represents the Q-criterion, and the red iso-surfaces represent the gas temperature of $1800 \mathrm{~K}$. This figure shows the complexity of the spray-turbulence-flame interaction in the shear flow.

The impact of the jet velocity on the ignition process can be explained by the temporal evolution of the volume-averaged temperature, as it seen from Figure 3. Increasing the jet velocity decreases the ignition delay time and increases the volume-averaged temperature. Consequently, the evaporation (source of temperature reduction) is completed faster for higher jet velocity.

Figure 3. Volume-averaged Temperature versus Time for Different Values of the Jet Velocity

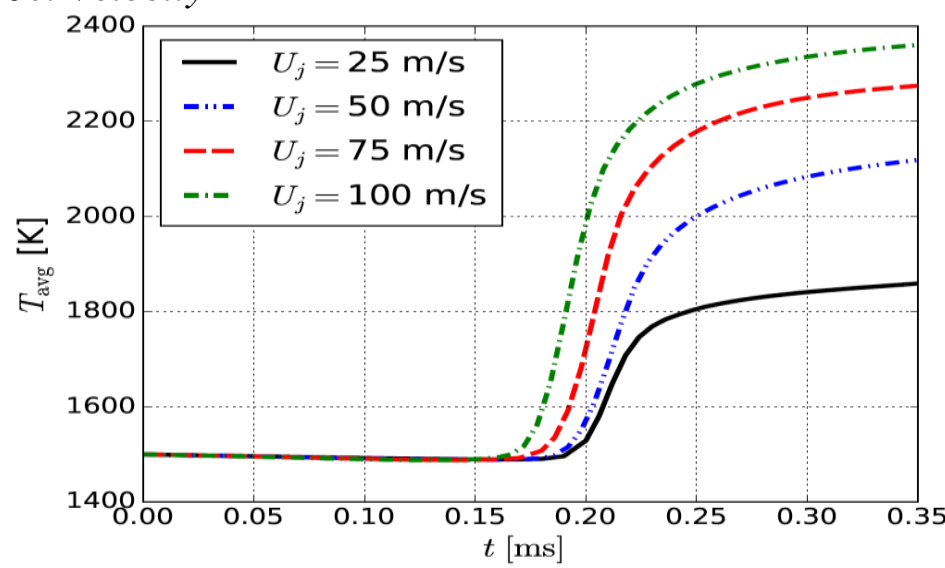

Similarly, Figure 4 shows the temporal evolution of the volume-averaged scalar dissipation rate versus time. As it is observed, the volume-averaged value decreases monotonically with the jet velocity. This can be explained by the previous figure (Figure 3); in case of higher velocity, the dilatation becomes stronger due to the faster evaporation and ignition process (high temperature).

Figure 4. Volume-averaged Scalar Dissipation Rate versus Time for Different Values of the Jet Velocity

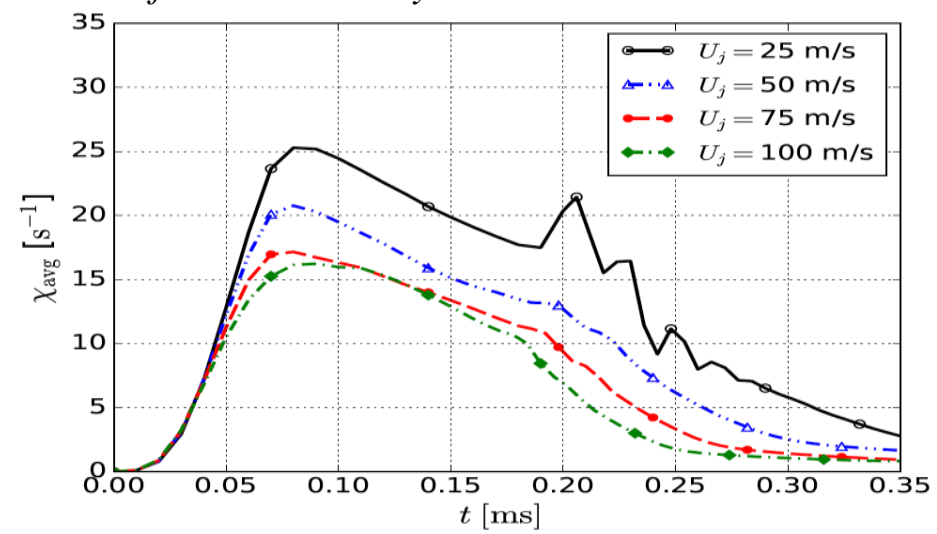


Vol. 4, No. $4 \quad$ Abdelsamie et al.: Direct Numerical Simulations of Turbulent Spray...

Figure 5 depicts the scatter plot of the scalar dissipation rate versus the mixture fraction. As it is seen from this figure, the maximum values of scalar dissipation rate are found near to the stoichiometric mixture $\left(\zeta_{\mathrm{z}, \mathrm{st}}=0.062\right.$ for the $\mathrm{n}$ heptane/air mixture) for all cases. In the case with $U_{j}=25 \mathrm{~m} / \mathrm{s}, \chi$ shows a specific behavior, with high values of $\chi$ found over a wide range of mixture fraction, also on the rich side.

Figure 5. Scatter Plot of Scalar Dissipation Rate versus Mixture Fraction at Ignition Time. (a) $U_{j}=25 \mathrm{~m} / \mathrm{s}$, (b) $U_{j}=50 \mathrm{~m} / \mathrm{s}$, (c) $U_{j}=75 \mathrm{~m} / \mathrm{s}$, (d) $U_{j}=100 \mathrm{~m} / \mathrm{s}$.

(a)

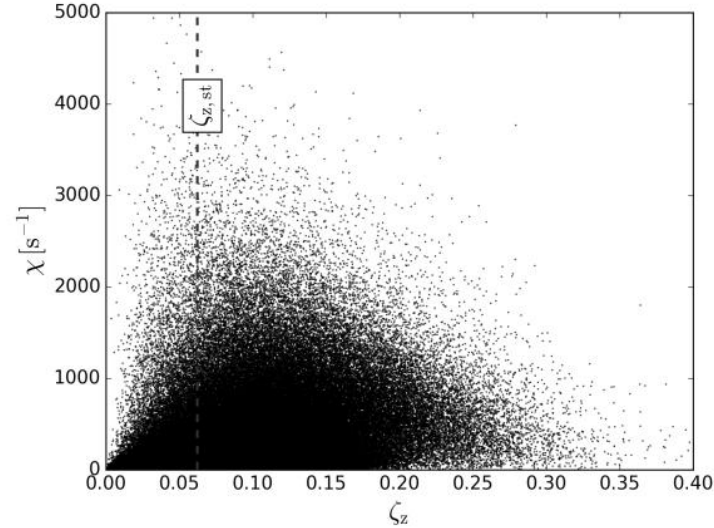

(b)

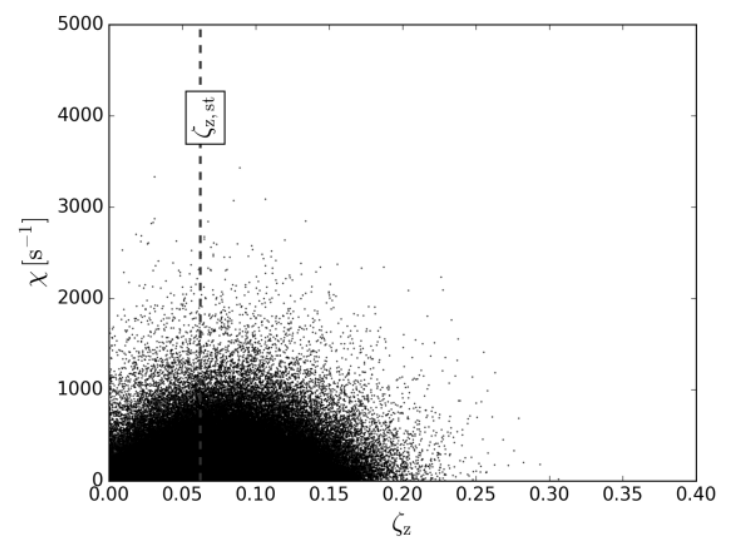

(c)

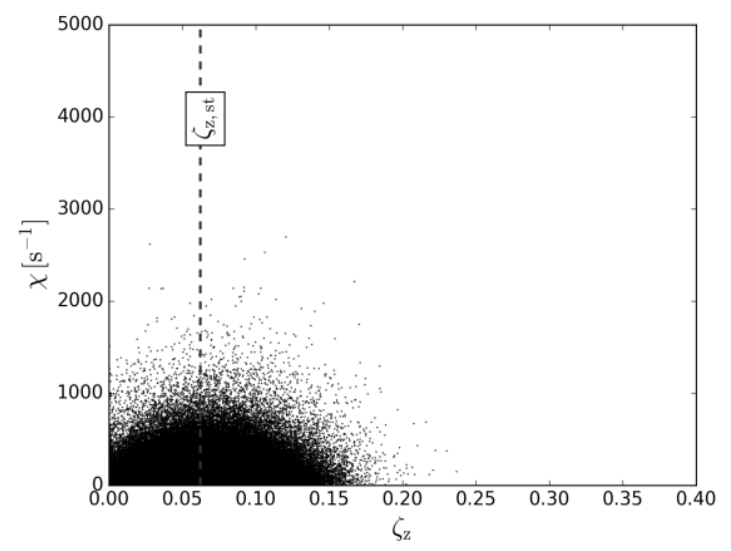


(d)

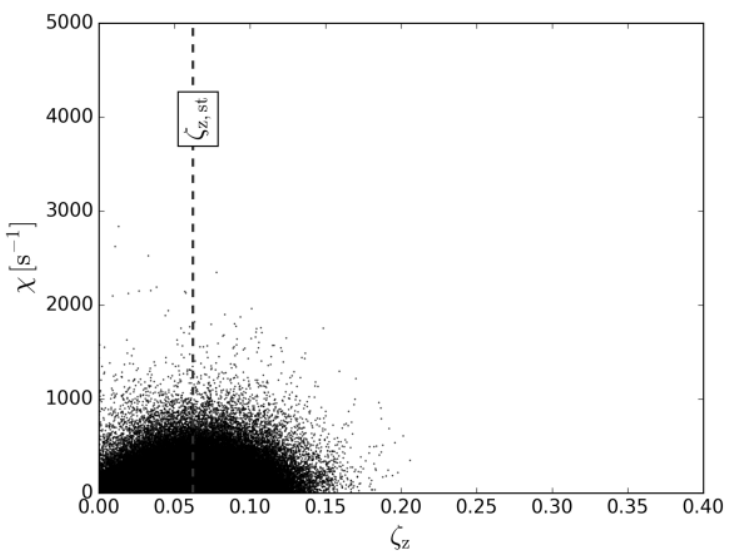

When the jet velocity increases, the maximum value of $\chi$ decreases and is found for a diminishing range of mixture fraction. This behavior can be examined using Figure 6. This figure shows the conditional mean of scalar dissipation rate $\left\langle\chi \mid \zeta_{z}\right\rangle$ versus the mixture fraction at ignition time for different jet velocities. At low velocity, $U_{j}=25 \mathrm{~m} / \mathrm{s},\left\langle\chi \mid \zeta_{z}\right\rangle$ shows the highest values over all mixture fraction values; this is associated to an increased ignition delay time, allowing for more evaporation but less dilatation, leading to larger scalar dissipation rates. Increasing the jet velocity (shear) decreases $\left\langle\chi \mid \zeta_{z}\right\rangle$ at $\zeta_{z} \leq \zeta_{z, s t}$, for similar reasons. However, for $\left.\zeta_{z}\right\rangle \zeta_{z, s t},\left\langle\chi \mid \zeta_{z}\right\rangle$ slightly increases with jet velocity, as a consequence of improved mixing but at relatively low temperatures in this rich region.

Figure 6. Conditional Mean of Scalar Dissipation Rate versus Mixture Fraction at Ignition Time for Different Jet Velocities

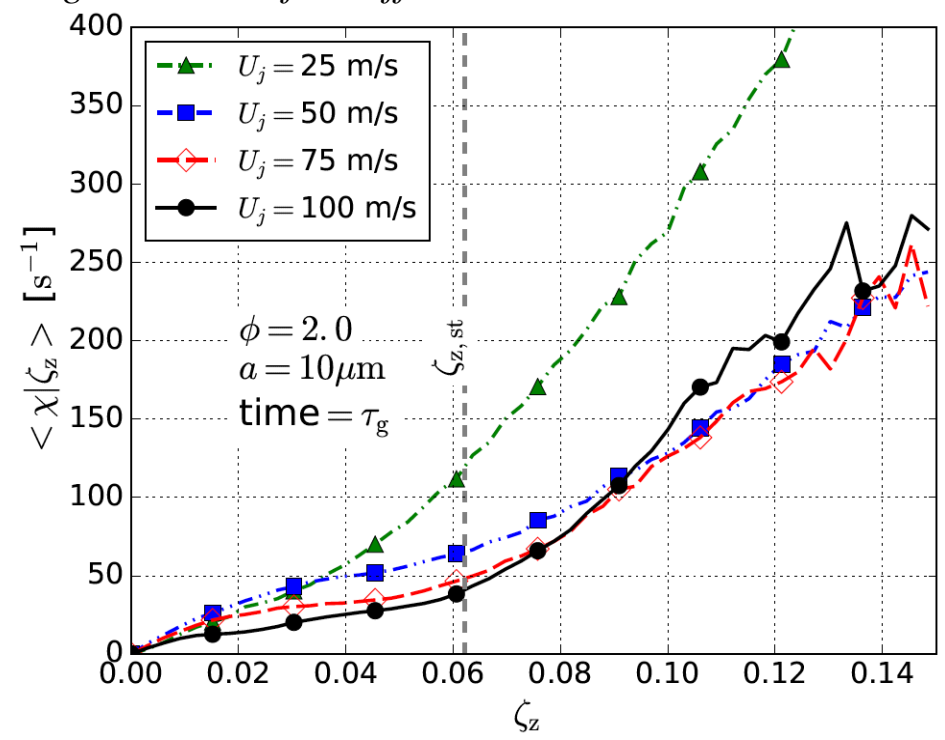

Figures 5 and 6 demonstrate the complexity of the scalar dissipation rate behavior in spray turbulent combustion, leading to a considerable modeling challenge, since $\left\langle\chi \mid \zeta_{z}\right\rangle$ shows a non-monotonic behavior in the mixture fraction 
space. Therefore, it is recommended to examine turbulent spray combustion with reliable models (here, DNS with detailed chemistry), and for different shear values, in order to derive generally applicable models.

\section{Conclusions}

In this work, 3D DNS-DPS simulations have been performed to investigate the behavior of the scalar dissipation rate in turbulent spray combustion. A spray in a temporally-evolving jet configuration has been chosen for this investigation. Four different cases have been studied by changing the jet velocity $\left(U_{j}=25,50,75,100 \mathrm{~m} / \mathrm{s}\right)$. The results show that increasing the jet velocity decreases the ignition delay time and increases the volume-averaged temperature. Consequently, the evaporation is completed faster for higher jet velocity. It is also observed that, the volume-averaged of scalar dissipation rate decreases monotonically with the jet velocity; this could be explained as in case of higher velocity, the dilatation becomes stronger due to the faster evaporation and ignition process (high temperature). Locally, the scatter plots illustrate that when the jet speed increases, the maximum value of scalar dissipation rate $\chi$ decreases and is found for a diminishing range of mixture fraction. Moreover, the conditional averaged value of scalar dissipation rate shows non-monotonic behavior over the mixture fraction space; as consequence, developing generally valid models will be difficult. This study shows how future simulations (under different conditions) will help develop a database supporting model development.

\section{Acknowledgments}

The financial support of the DFG (Deutsche Forschungsgemeinschaft) for Abouelmagd Abdelsamie within the project FOR1447 "Physicochemical-based models for the prediction of safety-relevant ignition processes" is gratefully acknowledged. The computer resources provided by the Gauss Center for Supercomputing/Leibniz Supercomputing Center Munich under grant pro84qo have been essential to obtain the results presented in this work. 


\section{Nomenclature}

$a_{d} \quad$ diameter of the droplet $d$

$\mathrm{B}_{T, d} \quad$ heat transfer number

$\mathrm{B}_{m} \quad$ Spalding mass transfer number

$C_{p} \quad$ specific heat at constant pressure

$C_{p, k} \quad$ specific heat of species $k$ at constant pressure

$C_{p, f}^{F} \quad$ specific heat of the fuel vapor in the film region

$C_{p, f} \quad$ specific heat of the gas mixture in the film region

$h_{k} \quad$ specific enthalpy of species $k$

$H \quad$ width of the planar jet

$L \quad$ domain length

$L_{v} \quad$ molar latent heat of droplet vaporization

$L_{v, s} \quad$ latent heat at the reference temperature

$N \quad$ total number of species

$\mathrm{Nu} \quad$ Nusselt number

$\tilde{p} \quad$ dynamic fluctuating pressure

$p_{\text {ref }} \quad$ reference (atmospheric) pressure

$p_{\text {sat }} \quad$ saturated vapor pressure

$p_{\infty} \quad$ thermodynamic pressure of gas mixture in the far-field

Pr Prandtl number

$\mathrm{R}_{f} \quad$ perfect gas constant

$\mathrm{Re}_{d} \quad$ droplet Reynolds number

Sc Schmidt number

Sh Sherwood number

$t \quad$ time

$T \quad$ temperature of the flow

$T_{d} \quad$ temperature of the liquid droplet surface

$T_{c r} \quad$ critical temperature of the fuel

$T_{\text {ref }} \quad$ reference (boiling) temperature

$T_{\infty} \quad$ temperature of the gas mixture in far-field

$u_{i} \quad i$-th component of the flow velocity

$U_{c o} \quad$ co-flow speed

$\boldsymbol{U}_{d} \quad$ velocity vector of the $d$-th droplet

$U_{j} \quad$ jet speed

$\boldsymbol{U}_{\infty} \quad$ velocity vector of the surrounding gas mixture at droplet location

$V_{i, k} \quad i$-th component of diffusion velocity of species $k$

$W_{F} \quad$ molar mass of the fuel

$W_{O} \quad$ oxidizer molar mass

$x_{i} \quad i$-th component of the location vector

$\boldsymbol{X}_{d} \quad$ droplet location vector

$Y_{F, \infty} \quad$ gaseous fuel mass fraction in far-field

$Y_{k} \quad$ mass fraction of species $k$

$Y_{s, d} \quad$ mass fraction of fuel vapor at the droplet surface 
Greek Symbols

$\delta_{i j} \quad$ Kronecker delta

$\Delta x \quad$ grid resolution in $\mathrm{x}$ direction

$\zeta_{z} \quad$ mixture fraction

$\lambda$ heat diffusivity coefficient

$\mu \quad$ dynamic viscosity of the gas mixture

$\rho$ density of the gas mixture

$\sigma_{j i} \quad$ viscous stress tensor of the flow

$\tau_{U, d}$ momentum relaxation time

$\tau_{a, d}$ evaporation delay

$\tau_{T, d} \quad$ heating delay

$\phi \quad$ equivalence ratio of the gas mixture

$\chi \quad$ scalar dissipation rate of the mixture fraction

$\dot{\omega}_{k} \quad$ net production rate of species $k$

\section{Subscript}

d refers to droplet

$f \quad$ refers to the properties in the film region

$k \quad$ refers to the species; where $k=1,2, \ldots, N$

st refers to the stoichiometric condition

0 refers to properties at initial conditions

$\infty \quad$ refers to the properties in the far-field

\section{References}

Abdelsamie, A. and Thévenin, D., 2017. 'Direct numerical simulation of spray evaporation and autoignition in a temporally-evolving jet', Proc. Combust. Inst. 36(2), 2493-2502.

Abdelsamie, A., Fru, G., Oster, T., Dietzsch, F., Janiga, G. and Thévenin, D., 2016. 'Towards direct numerical simulations of low-Mach number turbulent reacting and two-phase flows using immersed boundaries', Comput. Fluids 131, 123-141.

Abramzon, B. and Sirignano, W. A., 1989. 'Droplet vaporization model for spray combustion calculations', Int. J. Heat Mass Transfer 32(9), 1618-1618.

Borghesi, G., Mastorakos, E. and Cant, R. S., 2013. 'Complex chemistry DNS of nheptane spray autoignition at high pressure and intermediate temperature conditions', Combust. Flame 160, 1254-1275.

Domingo, P., Vervisch, L. and Réveillon, J., 2005. 'DNS analysis of partially premixed combustion in spray and gaseous turbulent flame-bases stabilized in hot air', Combust. Flame 140, 172-195.

Fréret, L., Thomine, O., Réveillon, J., de Chaisemartin, S., Laurent, F. and Massot, M., 2010. 'On the role of preferential segregation in flame dynamics in polydisperse evaporating sprays', In Proceedings of the Summer Program 2010, Center for Turbulence Research. Stanford University, California, USA, 2011, pp. 383-392.

Fujita, A., Watanabe, H., Kurose, R. and Komori, S., 2013. 'Two-dimensional direct numerical simulation of spray flames-Part 1: Effects of equivalence ratio, fuel droplet size and radiation, and validity of flamelet model', Fuel 104,515-525. 
Jin, T., Luo, K., Dai, Q. and Fan, J., 2016. 'Direct numerical simulation on supersonic turbulent reacting and non-reacting spray jet in heated coflow', Fuel, 164, 267-276.

Kitano, T., Nakatani, T., Kurose, R. and Komori, S., 2013. 'Two-dimensional direct numerical simulation of spray flames-Part 2: Effects of ambient pressure and lift, and validity of flamelet model', Fuel 104,526-535.

Kitano, T., Nishio, J., Kurose, R. and Komori, S., 2014. 'Effects of ambient pressure, gas temperature and combustion reaction on droplet evaporation', Combust. Flame 161, 551-564.

Mashayek, F., 2000. 'Numerical investigation of reacting droplets in homogeneous shear turbulence', J. Fluid Mech. 405, 1-36.

Neophytou, A., Mastorakos, E. and Cant, R. S., 2011. 'Complex chemistry simulations of spark ignition in turbulent sprays', Proc. Combust. Inst. 33, 2135-2142.

Neophytou, A., Mastorakos, E. and Cant, R. S., 2012. 'The internal structure of igniting turbulent sprays as revealed by complex chemistry DNS', Combust. Flame 159, 641664.

Patel, A., Kong, S. C. and Reitz, R. D., 2004. 'Development and validation of a reduced reaction mechanism for HCCI engine simulations', SAE Technical Paper, 2004-01055 .

Réveillon, J. and Vervisch, L., 2005. 'Analysis of weakly turbulent dilute-spray flames and spray combustion regimes', J. Fluid Mech. 537, 317-347.

Réveillon, J. and Demoulin, F. X., 2007. 'Evaporating droplets in turbulent reacting flows', Proc. Combust. Inst. 31, 2319-2326.

Réveillon, J., Pera, C. and Bouali, Z., 2011. 'Examples of the potential of DNS for the understanding of reactive multiphase flows', Int. J. Spray Combust. Dyn. 3(1), 63-92.

Wandel, A. P. , Chakraborty, N. and Mastorakos E., 2009. 'Direct numerical simulations of turbulent flame expansion in fine sprays', Proc. Combust. Inst. 32, 2283-2290.

Wang, Y. and Rutland, C. J., 2005. 'Effects of temperature and equivalence ratio on the ignition of n-heptane fuel spray in turbulent flow', Proc. Combust. Inst. 30, 893-900, 2005.

Wang, Y. and Rutland, C. J., 2007. "Direct numerical simulation of ignition in turbulent nheptane liquid-fuel spray jets", Combust. Flame 149, 353-365.

Wang, H., Luo, K. and Fan, J., 2014. 'Effects of turbulent intensity and droplet diameter on spray combustion using direct numerical simulation', Fuel 121, 311-318. 
